\title{
Constraints on millicharged dark matter and axionlike particles from timing of radio waves
}

\author{
Andrea Caputo, ${ }^{1}$ Laura Sberna $\odot,{ }^{2, *}$ Miguel Frías, ${ }^{3}$ Diego Blas, ${ }^{4}$ Paolo Pani, ${ }^{5}$ Lijing Shao, ${ }^{6}$ and Wenming Yan $^{7}$ \\ ${ }^{1}$ Instituto de Física Corpuscular, Universidad de Valencia and CSIC, Edificio Institutos Investigación, \\ Catedrático Jose Beltrán 2, Paterna, 46980 Spain \\ ${ }^{2}$ Perimeter Institute, 31 Caroline St N, Ontario, Canada \\ ${ }^{3}$ Facultat de Física, Universitat de Barcelona, Martí Franquès 1, 08028 Barcelona, Catalonia, Spain \\ ${ }^{4}$ Theoretical Particle Physics and Cosmology Group, Department of Physics, King's College London, \\ Strand, London WC2R 2LS, United Kingdom \\ ${ }^{5}$ Dipartimento di Fisica, "Sapienza" Università di Roma \& Sezione INFN Romal, \\ Piazzale Aldo Moro 5, 00185, Roma, Italy \\ ${ }^{6}$ Kavli Institute for Astronomy and Astrophysics, Peking University, Beijing 100871, China \\ ${ }^{7}$ Xinjiang Astronomical Observatory, CAS, 150 Science 1-Street, Urumqi, Xinjiang 830011, China
}

(Received 15 February 2019; published 16 September 2019)

\begin{abstract}
We derive constraints on millicharged dark matter and axionlike particles using pulsar timing and fast radio burst observations. For dark matter particles of charge $\epsilon e$, the constraint from time of arrival (TOA) of waves is $\epsilon / m_{\text {milli }} \lesssim 10^{-8} \mathrm{eV}^{-1}$, for masses $m_{\text {milli }} \gtrsim 10^{-6} \mathrm{eV}$. For axionlike particles, the polarization of the signals from pulsars yields a bound in the axial coupling $g / m_{a} \lesssim 10^{-13} \mathrm{GeV}^{-1} /\left(10^{-22} \mathrm{eV}\right)$, for $m_{a} \lesssim 10^{-19} \mathrm{eV}$. Both bounds scale as $\left(\rho / \rho_{\mathrm{dm}}\right)^{1 / 2}$ for fractions of the total dark matter energy density $\rho_{\mathrm{dm}}$. We make a precise study of these bounds using TOA from several pulsars, FRB 121102, and polarization measurements of PSR J0437 - 4715. Our results rule out a new region of the parameter space for these dark matter models.
\end{abstract}

DOI: $10.1103 /$ PhysRevD.100.063515

\section{INTRODUCTION}

Unraveling the nature of dark matter (DMa) is among the most urgent issues in fundamental physics. Indirect searches aim at detecting the effects of DMa in astrophysical observations, beyond its pure gravitational interaction. Given the feeble interaction of DMa with standard model fields, precise measurements are particularly promising for these searches. When one requires precision, a particular measurement stands out in astrophysics: the time of arrival (TOA) of radio waves from pulsars and fast radio bursts (FRBs). The use of pulsar timing has already been suggested to study the effects of dark matter [1-9]. In this paper, we present new results for DMa models directly coupled to light from the propagation of radio pulses from pulsars and FRBs. A more comprehensive exploration will be presented elsewhere [10].

If DMa is coupled to the electromagnetic field, one expects modifications in the emission, propagation, and detection of radio pulses. We focus here on the effects during the propagation, which are robust under astrophysical uncertainties. In particular, we derive stringent constraints on millicharged DMa and axionlike particles (ALPs) based on dispersion measurements (DMs) of radio signals from pulsars and FRBs, and on the modulation of

*Corresponding author. 1sberna@perimeterinstitute.ca the light polarization angle due to axionlike DMa in the Milky Way.

We give a unified treatment, where the millicharged DMa and ALPs are considered as independent species. In the former case, we consider that (a fraction of) the DMa is made of particles with mass $m_{\text {milli }}$ and electric charge $q=\epsilon e \quad(\epsilon \ll 1)$ [11-18]. As an example, this coupling arises in models where the DMa is charged under a dark photon, which is kinematically coupled to the visible photon [16,17]. In our analysis, we remain agnostic to the origin of this term and other possible model-dependent signatures behind the charge of the DMa, and focus on constraining $\epsilon$. Regarding ALPs, we assume the existence of axionlike [19-23], pseudoscalar DMa of mass $m_{a}$ (represented by the field $\phi$ below).

The relevant field equations read

$$
\begin{gathered}
\left(\square-m_{a}^{2}\right) \phi=-\frac{g}{4} F_{\mu \nu} \tilde{F}^{\mu \nu}, \\
\partial_{\mu} F^{\mu \nu}=4 \pi e j^{\nu}+4 \pi \epsilon e j_{\text {milli }}^{\nu}-\frac{g}{2} \epsilon^{\mu \rho \lambda \nu} F_{\mu \rho} \partial_{\lambda} \phi,
\end{gathered}
$$

where $g$ is the ALP-photon axial coupling, $j^{\nu}$ is the ordinary electron current, whereas $j_{\text {milli }}^{\nu}$ is the current from millicharged particles. The role of this term in the propagation of radio waves will be studied in the next section, under the 
assumption of a cold distribution of the millicharge DMa component.

\section{DISPERSION IN THE TOA}

We consider the propagation of a light signal of frequency $\nu=\omega /(2 \pi)$ along the $z$ direction in the presence of a homogeneous background magnetic field polarized along (say) the $y$ direction, $\vec{B}=(0, B, 0)$. We neglect a possible $B_{z}$ component in this section since its role in dispersion of the light signal in a medium of particles of mass $m_{q}$ and charge $q$ is suppressed by $q B_{z} /\left(m_{q} \omega\right)$, always small for the cases we study. For the first part of this work, DMa is considered as a cold medium with vanishing background values for the fields appearing in (1) and (2). When $\omega \gg m_{a}$, the propagation of the light signal in this medium is described by the first-order system $i \frac{\partial}{\partial z}|\psi(z)\rangle=\mathcal{M}|\psi(z)\rangle$, where the $|\psi(z)\rangle$ is a linear combination of the two photon polarizations along the $x$ and $y$ directions and of the ALP state [24]. The $3 \times 3$ mixing matrix reads [25]

$$
\mathcal{M}:=\left(\begin{array}{ccc}
\omega+\Delta_{x x} & 0 & 0 \\
0 & \omega+\Delta_{y y} & g B / 2 \\
0 & g B / 2 & \omega-m_{a}^{2} /(2 \omega)
\end{array}\right) .
$$

The terms $\Delta_{x x}$ and $\Delta_{y y}$ contain both QED vacuum polarization effects and plasma effects [24,25]. The first ones are of order $\Delta_{x x}^{\mathrm{QED}} \sim \Delta_{y y}^{\mathrm{QED}} \sim \omega \frac{e^{2}}{45 \pi}\left(\frac{B}{B_{c}}\right)^{2}$, where $B_{c} \approx 4 \times 10^{13} \mathrm{G}$ [26]. We shall only consider interstellar magnetic fields, for which $B \ll B_{c}$ and $\Delta^{\mathrm{QED}}$ effects are negligible. Plasma effects arise from the presence of free charges. In the limit where the photon energy is much smaller than the mass of the charged, cold particles [27-29],

$$
\Delta_{x x}^{\text {plasma }} \sim \Delta_{y y}^{\text {plasma }} \sim-\frac{\omega_{p}^{2}}{2 \omega},
$$

where $\omega_{p}^{2}:=\sum_{i} \frac{4 \pi n_{i} q_{i}^{2}}{m_{i}}$ is the plasma frequency for particles with charge $q_{i}$, mass $m_{i}$, and number density $n_{i}$. The normal modes corresponding to (3) satisfy

$k_{0}=\omega-\frac{\omega_{p}^{2}}{2 \omega}, \quad k_{ \pm}=\frac{4 \omega^{2}-\omega_{p}^{2}-m_{a}^{2} \mp \sqrt{\Delta_{\omega}}}{4 \omega}$,

with $\Delta_{\omega}=\left(m_{a}^{2}-\omega_{p}^{2}\right)^{2}+4 B^{2} g^{2} \omega^{2}$. The last term in $\Delta_{\omega}$ is always subdominant and we treat it perturbatively.

The TOA of a signal traveling at speed $v=\partial \omega / \partial k$ across a distance $d$ is $T=\int_{0}^{d} \frac{\mathrm{d} l}{v}=\int_{0}^{d} \mathrm{~d} l \frac{\partial k}{\partial \omega}$ along the line of sight. From the previous expressions, one finds for the relevant polarizations

$$
v_{0}^{-1}=1+\frac{\omega_{p}^{2}}{2 \omega^{2}},
$$

$$
v_{-}^{-1}=v_{0}^{-1}+\frac{B^{2} g^{2}}{2\left(m_{a}^{2}-\omega_{p}^{2}\right)}-\frac{3 B^{4} g^{4} \omega^{2}}{2\left(m_{a}^{2}-\omega_{p}^{2}\right)^{3}} .
$$

In the absence of new physics $(\epsilon=g=0)$, the previous modes propagate with velocity $v_{0}$. For a photon with frequency $\nu$, a background of cold free electrons yields a time delay

$$
\begin{aligned}
\Delta t_{\mathrm{DM}}^{\text {astro }} & =\frac{1}{2 \pi} \frac{e^{2}}{m_{e}} \mathrm{DM}_{\text {astro }}\left(\nu^{-2}-\nu_{\infty}^{-2}\right) \\
& \sim 4.15\left(\frac{\mathrm{DM}_{\mathrm{astro}}}{\mathrm{pc} \mathrm{cm}^{-3}}\right)\left(\frac{\nu}{\mathrm{GHz}}\right)^{-2} \mathrm{~ms},
\end{aligned}
$$

relative to a photon with high enough energy $\left(\nu_{\infty}\right.$ in the previous formula) [30]. Here $\mathrm{DM}_{\text {astro }}:=\int n_{e} \mathrm{~d} l$ is the standard dispersion measure (DM) from electrons with number density $n_{e}$ along the light of sight. The last line is also the observational definition of the dispersion measure, $\mathrm{DM}_{\mathrm{obs}}$. Comparing this number with the ALP-photon coupling term in Eq. (7), one sees that the modifications from the interstellar or intergalactic magnetic fields $(B \lesssim \mu \mathrm{G})$ are only relevant for $g>\mathrm{GeV}^{-1}$, which is already excluded by other methods, e.g., [31]. We ignore these terms in the following. We have checked that the high magnetic field of the pulsar magnetosphere is also not relevant for our studies and we ignore it. Finally, the local conditions of FRBs are not known. It is rather unlikely that they play a role in the DM and even more that they cancel the effects from the DMa plasma, Eqs. (6) and (7). We hence restrict our analysis of the TOA to the millicharged DMa.

\section{TOA CONSTRAINTS ON MILLICHARGED DMa}

As we explained above, we now focus on the case of millicharged DMa, i.e., $g=0$. The contribution of the millicharged DMa to the time delay is given by an expression analogue to (8), now considering the DMa particles as the dispersive medium,

$$
\Delta t_{\mathrm{DM}}^{\text {milli }}=\frac{1}{2 \pi} \frac{\epsilon^{2} e^{2}}{m_{\text {milli }}} \int \mathrm{d} l n_{\text {milli }}\left(\nu^{-2}-\nu_{\infty}^{-2}\right) .
$$

In this case, the observed DM is dominated by the sum of the contributions from ordinary electrons and millicharged particles (see also [32]), $\mathrm{DM}_{\mathrm{obs}}=\mathrm{DM}_{\text {astro }}+\mathrm{DM}_{\text {milli }}$, where the millicharged contribution is obtained by comparing (8) and (9),

$$
\mathrm{DM}_{\text {milli }}=\left(\frac{\epsilon}{m_{\text {milli }}}\right)^{2} m_{e} \int \mathrm{d} l \rho_{\text {milli }}
$$

where $\rho_{\text {milli }}$ is the density of millicharged particles, which is equal to or smaller than the full DMa density $\rho_{\mathrm{dm}}$. While the effect of $\mathrm{DM}_{\text {astro }}$ and $\mathrm{DM}_{\text {milli }}$ is completely degenerate, for a 
source at a distance $d$ any measurement of the DM can be translated into a conservative upper bound on $\epsilon / m_{\text {milli }}$ by simply requiring that all the DM is due to DMa, i.e., $\mathrm{DM}_{\text {milli }}<\mathrm{DM}_{\mathrm{obs}}$. This yields

$\frac{\epsilon}{m_{\text {milli }}} \lesssim \frac{10^{-8}}{\mathrm{eV}} \sqrt{\frac{0.3 \mathrm{GeV} / \mathrm{cm}^{3}}{\rho_{\text {milli }}}} \sqrt{\frac{\mathrm{DM}_{\mathrm{obs}}}{20 \mathrm{pc} / \mathrm{cm}^{3}}} \sqrt{\frac{400 \mathrm{pc}}{d}}$,

where we normalized the quantities by typical values within the galaxy. This estimate gives already a rather stringent bound, which can be refined through a Bayesian analysis. In the following, we closely follow [33]. Given our theoretical hypothesis $\left(\mathrm{DM}_{\mathrm{obs}}=\mathrm{DM}_{\mathrm{astro}}+\mathrm{DM}_{\text {milli }}\right)$, and the set of measurements of $\mathrm{DM}_{\mathrm{obs}}$ from $N$ pulsars, we construct the log likelihood as

$$
\ln \mathcal{L}=-\frac{1}{2} \sum_{i=1}^{N} \frac{\left(\mathrm{DM}_{\mathrm{obs}}^{i}-\mathrm{DM}_{\mathrm{astro}}^{i}-\mathrm{DM}_{\text {milli }}^{i}\right)^{2}}{\sigma_{i}^{2}} .
$$

Here $\sigma_{i}$ is the dispersion for each pulsar, obtained adding in quadrature statistical uncertainties on $\mathrm{DM}_{\mathrm{obs}}^{i}$ and the astrophysical ones on $\mathrm{DM}_{\mathrm{astro}}^{i}$. We used a uniform prior on $\epsilon / m_{\text {milli }}>0$ and verified that our results do not depend on this choice.

We shall consider two datasets of pulsars extracted from the ATNF Pulsar Catalogue [34], as explained in the Appendix. In both cases, we assume a Navarro-FrenkWhite (NFW) profile for the DMa density, normalized to a local value of $\rho_{\mathrm{dm}} \approx 0.3 \mathrm{GeV} / \mathrm{cm}^{3}$. The first dataset comprises $N=13$ local pulsars with the smallest values of $\mathrm{DM}_{\mathrm{obs}} / d$ and for which parallax measurements of the distance $d$ are available. We only choose pulsars located away from the galactic plane. This is to minimize the effect of the evacuation of DMa from the galactic plane for millicharged DMa. While early studies argue that this effect is relevant for $\epsilon \gtrsim 5.4 \times 10^{-22}\left(\frac{m_{\text {milli }}}{\mathrm{eV}}\right)[16,35]$, a recent study [36] suggests that this bound may be too restrictive. We also consider a second dataset of $N_{\text {cluster }}=13$ pulsars located in globular clusters within $8 \mathrm{kpc}$ from the galactic center and off the disk, again with the smallest $\mathrm{DM}_{\mathrm{obs}} / d$. Distances of clusters can be determined by different methods [37] not relying on the DM, and their uncertainty is usually of a few percent. We therefore assign a conservative error of $10 \%$ to the value of $d$ for the pulsars in this second dataset. Even if the effect of the galactic magnetic field on the density of millicharged DMa away from the galactic disk is uncertain, we do not expect DMa to be evacuated at high galactic latitudes, and our analysis should provide realistic constraints.

For each pulsar, we compute $\mathrm{DM}_{\text {astro }}^{i} \approx\left\langle n_{e}\right\rangle_{i} d_{i}$, where $\left\langle n_{e}\right\rangle_{i}$ is an average electron density along the line of sight obtained using the YMW16 model [38], while $d_{i}$ is the pulsar distance obtained from parallax (for the first dataset) or from the location of the globular cluster (for the second dataset). In the former case, we assign $\left\langle n_{e}\right\rangle_{i}$ a $20 \%$ error to

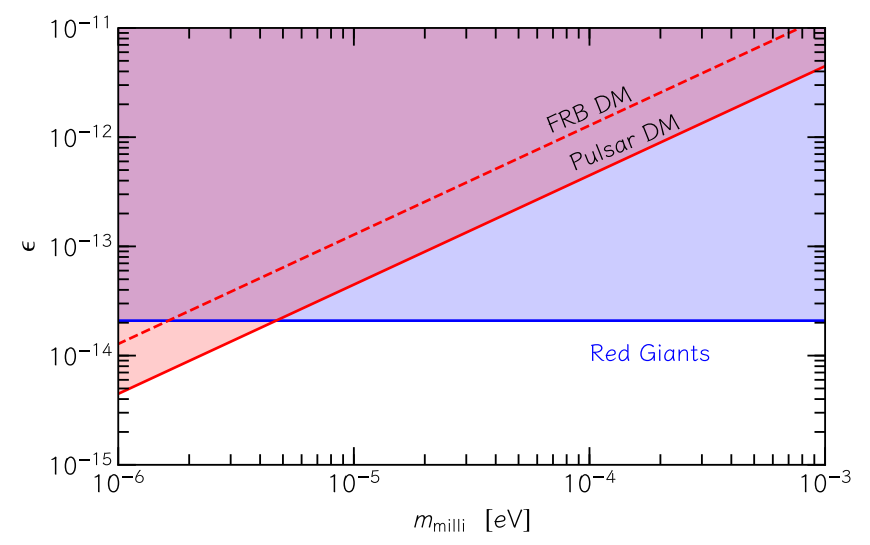

FIG. 1. Constraint on millicharged DMa in the $\epsilon-m_{\text {milli }}$ space from pulsar (solid red line) and FRB 121102 (dashed red line) $\mathrm{DM}$ at $95 \%$ confidence level. Solid blue line indicates the bound from Red Giants [15]. We assume a homogeneous DMa density $\rho_{\mathrm{dm}}=\rho_{\text {milli }} \approx 0.3 \mathrm{GeV} / \mathrm{cm}^{3}$. The bound scales as $\rho_{\text {milli }}^{-1 / 2}$ for fractional components.

take into account potential systematics in the electron density model. This is a conservative approach given the uncertainties in [38]. We perform a Monte Carlo Markov chain analysis using the PYTHON ensemble sampler EMCEE [39] to explore the posterior distribution. For our datasets, $10^{5}$ samples are accumulated with 20 chains. The chains show good acceptance rate and convergence. The results are similar for the two datasets

$\frac{\epsilon}{m_{\text {milli }}} \lesssim \frac{4 \times 10^{-9}}{\mathrm{eV}} \sqrt{\frac{0.3 \mathrm{GeV} / \mathrm{cm}^{3}}{\rho_{\text {milli }}}}$ at $95 \%$ C.L.,

which we compare to other existing bounds in Fig. 1. In particular, these results are compatible with $\epsilon=0$. For completeness, we also show a similar (weaker) bound estimated from the dispersion of the fast radio burst FRB 121102 [40]. This line falls in the ballpark of the estimate (11). A more comprehensive analysis for FRBs will be presented elsewhere [10].

The mass range in Fig. 1 is limited on the left because the expression (4) is valid as long as the energy of the photon is smaller than $m_{\text {milli }}$. For radio waves from pulsars, $m_{\text {milli }} \gtrsim \omega \sim \mathrm{GHz} \sim 10^{-6} \mathrm{eV}$. Since the bound is more stringent for small masses, these constraints could improve as $1 / m_{\text {milli }}$ for sub-GHz pulsar measurements in systems with properties similar to the ones used in our analysis. Low-frequency measurements are indeed possible; see, e.g., Ref. [41], though we leave a more systematic study of the sources for the future. Figure 1 shows that our bounds are competitive for masses below the TremaineGunn bound on fermionic DMa, $m_{\mathrm{TG}} \gtrsim \mathrm{KeV}$ [42]. Hence, they apply to scalar charged DMa or to models with a fraction of millicharged fermionic DMa [see Eq. (13) for the scaling of the bound with $\rho_{\text {milli }}$. 
Finally, the existence of millicharged DMa also impacts the cosmological $21-\mathrm{cm}$ line and distortions of the CMB [43-45]. It seems possible that these observations also constrain the very light case considered here, though previous studies focus on much heavier DMa candidates, and it seems cautious not to extrapolate their conclusions at much lower masses. Instead, it would be interesting to extend these analyses to smaller masses in the future.

\section{POLARIZATION CONSTRAINTS ON ALPS}

We now consider the case where the millicharged particles are absent, $j_{\text {milli }}^{\nu}=0$. As discussed before, the modification of the TOA from the terms depending on $g$ in Eq. (7) is negligible and we ignore it. Nevertheless, due to their pseudoscalar nature, ALPs also induce an oscillating variation of light polarization [46-52]. Parity-symmetry breaking leads to birefringence, i.e., different phase velocities for left- and right-handed modes, which in turn induces rotation of the linear polarization plane. At first approximation, we assume the ALP-DMa background in the Milky Way rest frame to be described by the field configuration [53]

$$
\phi(x, t)=\tilde{\phi}_{0}(x) \int \mathrm{d}^{3} v e^{-\frac{v^{2}}{\sigma_{0}^{2}}} e^{i\left(\omega_{v} t-m_{a} \vec{v} \cdot \vec{x}\right)+i \varphi_{\mathrm{v}}}+\text { c.c. },
$$

where $\sigma_{0} \approx 10^{-3}$ corresponds to the virialized velocity of the Milky Way and $\varphi_{\mathbf{v}}$ are arbitrary phases. The value $\tilde{\phi}_{0}$ changes smoothly with $x$ to reproduce the DMa energy density. Finally, for this nonrelativistic configuration, one can assume that $\omega_{v} \approx m_{a}\left(1+v^{2} / 2\right)$. For low DMa masses, this field configuration has only long modes as compared to the wavelength of radio signals and an eikonal approximation can be used to study the propagations of waves in this continuous background [54]. The leading result of this calculation yields an effect for the polarization angle of a photon propagating from time $t$ to $t+T[46,47]$,

$$
\begin{aligned}
\theta(t, T) \sim & 1.4 \times 10^{-2} \sin \left(m_{a} t+\delta\right) \\
& \times\left(\frac{g}{10^{-12} \mathrm{GeV}^{-1}}\right) \frac{10^{-22} \mathrm{eV}}{m_{a}} \mathrm{rad}
\end{aligned}
$$

where $\delta$ is a phase over which we will marginalize. The characteristic time scale for the axion background oscillation is $T_{\mathrm{ALP}} \sim \frac{10^{-22} \mathrm{eV}}{m_{a}} \mathrm{y}$; if one continuously observes the polarized light from the source during a time $t_{\mathrm{obs}} \gtrsim T_{\mathrm{ALP}}$, the observed variation of the polarization angle (15) may constrain the amplitude of the axion oscillations, ${ }^{1}$ i.e., the coupling $g$ for a given mass $m_{a}$. Pulsars are observed for long

\footnotetext{
${ }^{1}$ Notice that after exploring a quarter of a period of oscillation, the original value of $\delta$ is not relevant. Hence, even if a system lives in a region with $\delta \ll 1$, the previous analysis is valid for masses satisfying $t_{\mathrm{obs}} \gtrsim T_{\mathrm{ALP}}$.
}

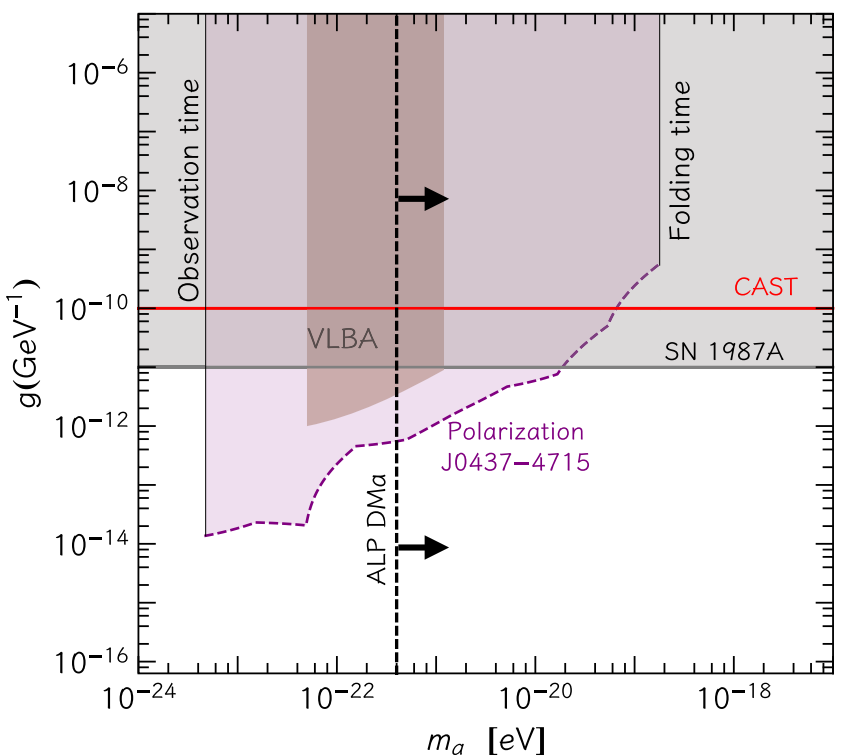

FIG. 2. Constraints for ALP DMa in the plane $g-m_{a}$ at 95\% C.L. The dash-dotted purple line indicates the lower bound set by polarization measurements using real data. The darker gray band indicates the region excluded by CAST experiment [31] and by supernova cooling [60], while the amaranth pink area indicates the region excluded by MOJAVE VLBA polarization observations of parsec-scale jets from active galaxies [46]. The vertical dashed line represents an estimation of the masses for which the ALP DMa candidate can constitute all the $\rho_{\mathrm{dm}}$ [57-59].

periods and the polarization angle is measured to be almost constant with a precision of roughly one degree, that can be compared with Eq. (15). We use the polarization data from Ref. [55] and in particular PSR J0437 - 4715, which is the pulsar with the highest number of observations of the polarization angle, spanning a period of roughly four years. The ionospheric contribution to the polarization angle was subtracted using the program GETRM-IONO [56]. Similar results are obtained when the ionospheric contribution is subtracted with the alternative FARROT method developed at the Dominion Radio Astrophysical Observatory, Penticton, Canada. We performed a likelihood estimation of the coupling $g$ for a set of fixed masses $m_{a}$. For each value of the mass, we marginalized over the unknown phase $\delta$ in Eq. (15) in the interval $[-\pi, \pi]$ and then obtained the 95\% C.L. exclusion value for $g$, which is our reported constraint. There is a caveat in using the bound from a single system: it may be that the pulsar of interest lives in a region where the amplitude of the field (14) is lower than expected from the NFW profile. This situation may happen, for instance, in certain ultralight DMa models where the field $\phi$ interpolates between different domains of condensation. The chances for this to happen are slim. Still, it is important to take this caveat into consideration. The use of more pulsars in the future will likely reduce this possibility even more.

The excluded region in Fig. 2 spans roughly 4 orders of magnitude in the mass range, from $m_{a} \sim 10^{-19} \mathrm{eV}$ to 
$m_{a} \sim 10^{-23} \mathrm{eV}$. The lower limit is set by the total observation time $(\sim 4 \mathrm{y})$, whereas the upper limit is set by the resolution time in the dataset during each observation run ("folding time"), that is roughly 1 hour for J0437 - 4715 . The derived lower bounds scale as $1 / m_{a}$-with some modulation due to the fact that observations of the polarization angle for J0437 - 4715 are not homogeneous in time-and are stronger for smaller masses, i.e., longer observation time. The bound scales as $\sim \sqrt{\rho_{\mathrm{dm}}}$, so it can be competitive even if ALPs form only a small fraction of the DMa. This is particularly important at low masses, where other astrophysical constraints require the mass of the ALP to be $m_{a} \gtrsim 10^{-21} \mathrm{eV}$ if it constitutes all the DMa. These bounds are based on the clustering properties of the DMa candidate at small scales [57], the modifications of rotation curves in the inner regions of galaxies [58], and the mere existence of galaxies with very small gravitational binding energies [59]. These constraints are subject to independent astrophysical uncertainties, though together they indicate that masses below $m_{a} \sim 10^{-21} \mathrm{eV}$ are in tension with current data. In Fig. 2, we represent the previous limit by a conservative line at $m_{a}=4 \times 10^{-21} \mathrm{eV}$. This limitation relaxes for fractional components.

\section{DISCUSSION}

Several DMa models introduce dispersion effects in the photon propagation. Although small, these effects accumulate for photons coming from astrophysical sources and can be constrained through precision measurements. The effect of millicharged DMa is degenerate with that of ordinary plasma and improving models for the local plasma distribution will help strengthening the constraints from DM. On the other hand, the effect of ALP-photon coupling is more striking and requires a careful analysis of the TOA as a function of the frequency. In addition, in the upcoming era of the Square Kilometre Array, we will benefit from a much larger pulsar sample (possibly comprising sources near the galactic center, where the DMa density is higher than what is assumed here), combined with a significantly improved timing precision [61-63]. The prospects of using radio waves in probing DMa are very promising in the near future. For ALPs, their coupling to photons generates an oscillation of the polarization angle of photons in the ultralight DMa case. Our results in Fig. 2 show that, for the mass range $10^{-23}-10^{-20} \mathrm{eV}$, the constraints derived here are the best available and will greatly improve in the future with more data.

We have considered propagation in a weak magnetic field for which dispersion due to the ALP-photon coupling and QED vacuum polarization effects are negligible. However, our formalism can be easily extended to include such effects, which might be relevant for propagation in strongly magnetized regions. A discussion of this effect will appear elsewhere [10].

\section{ACKNOWLEDGMENTS}

We are grateful to Nikita Blinov, Richard Brito, Anson Hook, Georg Raffelt, and Günter Sigl for interesting discussions. We thank Davide Racco and Mikhail M. Ivanov for pointing out a mistake in the first arXiv version of this work. A.C. acknowledges support from Spanish MINECO national Grants No. FPA2014-57816-P, No. FPA2017-85985-P, and the European Projects No. H2020-MSCAITN-2015//674896-ELUSIVES and No. H2020-MSCA-RISE2015. P. P. acknowledges financial support provided under the European Union's H2020 ERC, Starting Grant No. DarkGRA-757480, and support from the Amaldi Research Center funded by the MIUR program "Dipartimento di Eccellenza" (CUP: B81I18001170001) and by the GWverse COST Action CA16104, "Black holes, gravitational waves and fundamental physics." Research at Perimeter Institute is supported by the Government of Canada through Industry Canada and by the Province of Ontario through the Ministry of Research and Innovation. L. S. was partially supported by the National Science Foundation of China (Grant No. 11721303) and XDB23010200.

Note added.-While this work was close to completion, Ref. [64] appeared on the arXiv, estimating constraints on ALPs using the polarization angle of radio waves from pulsars similar to those derived in the second part of our work. Even though the idea is similar, our analysis, based on real data, is distinct and the results differ from the ones in [64] by roughly a factor $\sqrt{\frac{10^{-22} \mathrm{eV}}{m_{a}}} \sqrt{\frac{400 \mathrm{pc}}{d}}$ originating from a different assumption about the $\phi$ configuration.

\section{APPENDIX: PULSAR DM DATASETS}

We provide here additional details of the datasets analyzed in this work. For the millicharged DMa, we analyzed a first set of galactic pulsars selected for their minimal $\mathrm{DM} / d$, where $d$ is derived from parallax, and for their good agreement with the electron density model (Table I). A second set of pulsars is selected in galactic clusters (Table II). In this case, in addition to the aforementioned criteria, we also require that the pulsars are not further from the galactic center than the Solar System, $\sqrt{X^{2}+Y^{2}+Z^{2}}<8.3 \mathrm{kpc}$, and are also located far from the galactic disk, $|Z|>1 \mathrm{kpc}$. 
TABLE I. List of local pulsars considered in this work.

\begin{tabular}{lccc}
\hline \hline Pulsar & Parallax (mas) & DM $\left(\mathrm{pc} \mathrm{cm}^{-3}\right)$ & $n_{e}\left(\mathrm{~cm}^{-3}\right)$ \\
\hline $\mathrm{J} 1024-0719$ & $0.770 \pm 0.23$ & $6.4778 \pm 0.0006$ & 0.009036 \\
$\mathrm{~J} 1012+5307$ & $0.710 \pm 0.17$ & $9.02314 \pm 0.00007$ & 0.007827 \\
$\mathrm{~J} 2010-1323$ & $0.300 \pm 0.10$ & $22.177 \pm 0.005$ & 0.004931 \\
$\mathrm{~J} 2234+0611$ & $0.700 \pm 0.20$ & $10.7645 \pm 0.0015$ & 0.008292 \\
$\mathrm{~J} 1909-3744$ & $0.810 \pm 0.03$ & $10.3932 \pm 0.01$ & 0.016935 \\
$\mathrm{~B} 2020+28$ & $0.370 \pm 0.12$ & $24.63109 \pm 0.00018$ & 0.012689 \\
$\mathrm{~B} 1508+55$ & $0.470 \pm 0.03$ & $19.6191 \pm 0.0003$ & 0.004691 \\
$\mathrm{~J} 2017+0603$ & $0.400 \pm 0.20$ & $23.92344 \pm 0.00009$ & 0.011004 \\
$\mathrm{~B} 1534+12$ & $0.860 \pm 0.18$ & $11.61944 \pm 0.00002$ & 0.009608 \\
$\mathrm{~J} 0108-1431$ & $4.200 \pm 1.40$ & $2.38 \pm 0.19$ & 0.009246 \\
$\mathrm{~B} 0031-07$ & $0.930 \pm 0.08$ & $10.922 \pm 0.006$ & 0.008167 \\
$\mathrm{~J} 1023+0038$ & $0.731 \pm 0.022$ & $14.325 \pm 0.01$ & 0.008209 \\
$\mathrm{~B} 1237+25$ & $1.160 \pm 0.08$ & $9.25159 \pm 0.00053$ & 0.008940 \\
\hline \hline
\end{tabular}

TABLE II. List of pulsars in globular clusters considered in this work.

\begin{tabular}{lcccl}
\hline \hline Pulsar & Cluster & $d(\mathrm{pc})$ & $\mathrm{DM}(\mathrm{pc} \mathrm{cm}$ & \\
\hline B1516+02B & M5 & 8500 & $29.47 \pm 0.11$ & 0.000027 \\
B1516+02A & M5 & 8500 & $30.08 \pm 0.05$ & 0.000027 \\
J1518+ 0204D & M5 & 8000 & $29.3 \pm 0.11$ & 0.000042 \\
J1518+ 0204E & M5 & 8000 & $29.3 \pm 0.11$ & 0.000042 \\
J1518+ 0204C & M5 & 8000 & $29.3146 \pm 0.006$ & 0.000042 \\
J2140-2310A & M30 & 9200 & $25.0640 \pm 0.0041$ & 0.000015 \\
J2140-2310B & M30 & 9200 & $25.09 \pm 0.12$ & 0.000015 \\
J0024-7204X & 47Tuc & 4690 & $24.539 \pm 0.005$ & 0.0083 \\
J0024-7204Y & 47Tuc & 4690 & $24.45 \pm 0.01$ & 0.0083 \\
J0024-7204P & 47Tuc & 4690 & $24.29 \pm 0.03$ & 0.0083 \\
B0021-72H & 47Tuc & 4690 & $24.37 \pm 0.02$ & 0.0083 \\
B0021-72E & 47Tuc & 4690 & $24.236 \pm 0.002$ & 0.0083 \\
J0024-7204R & 47Tuc & 4690 & $24.361 \pm 0.007$ & 0.0083 \\
\hline \hline
\end{tabular}

[1] A. Khmelnitsky and V. Rubakov, J. Cosmol. Astropart. Phys. 02 (2014) 019.

[2] N. K. Porayko and K. A. Postnov, Phys. Rev. D 90, 062008 (2014).

[3] P. Pani, Phys. Rev. D 92, 123530 (2015).

[4] H. A. Clark, G. F. Lewis, and P. Scott, Mon. Not. R. Astron. Soc. 456, 1394 (2016); 464, 2468(E) (2017).

[5] D. Blas, D. L. Nacir, and S. Sibiryakov, Phys. Rev. Lett. 118, 261102 (2017).

[6] K. Schutz and A. Liu, Phys. Rev. D 95, 023002 (2017).

[7] I. De Martino, T. Broadhurst, S. H. H. Tye, T. Chiueh, H.-Y. Schive, and R. Lazkoz, Phys. Rev. Lett. 119, 221103 (2017).

[8] A. Caputo, J. Zavala, and D. Blas, Phys. Dark Universe 19, 1 (2018).

[9] J. A. Dror, H. Ramani, T. Trickle, and K. M. Zurek, Phys. Rev. D 100, 023003 (2019).

[10] D. Blas, A. Caputo, M. Frías, P. Pani, L. Sberna, L. Shao, and W. Yan, Probing dark matter with pulsar timing (to be published).

[11] A. De Rujula, S. L. Glashow, and U. Sarid, Nucl. Phys. B333, 173 (1990).

[12] M. L. Perl and E. R. Lee, Am. J. Phys. 65, 698 (1997).

[13] B. Holdom, Phys. Lett. B 166, 196 (1986).

[14] K. Sigurdson, M. Doran, A. Kurylov, R. R. Caldwell, and M. Kamionkowski, Phys. Rev. D 70, 083501 (2004); 73, 089903(E) (2006).

[15] S. Davidson, S. Hannestad, and G. Raffelt, J. High Energy Phys. 05 (2000) 003.

[16] S. D. McDermott, H.-B. Yu, and K. M. Zurek, Phys. Rev. D 83, 063509 (2011).

[17] A. Berlin, N. Blinov, G. Krnjaic, P. Schuster, and N. Toro, Phys. Rev. D 99, 075001 (2019).

[18] D. Ejlli, Phys. Rev. D 96, 023540 (2017).
[19] R. D. Peccei and H. R. Quinn, Phys. Rev. Lett. 38, 1440 (1977).

[20] S. Weinberg, Phys. Rev. 140, B516 (1965).

[21] J. Preskill, M. B. Wise, and F. Wilczek, Phys. Lett. 120B, 127 (1983).

[22] L. F. Abbott and P. Sikivie, Phys. Lett. 120B, 133 (1983).

[23] M. Dine and W. Fischler, Phys. Lett. 120B, 137 (1983).

[24] G. Raffelt and L. Stodolsky, Phys. Rev. D 37, 1237 (1988).

[25] A. Dupays, C. Rizzo, M. Roncadelli, and G. F. Bignami, Phys. Rev. Lett. 95, 211302 (2005).

[26] S. L. Adler, Ann. Phys. (N.Y.) 67, 599 (1971).

[27] M. Gell-Mann, M. L. Goldberger, and W. E. Thirring, Phys. Rev. 95, 1612 (1954).

[28] M. L. Goldberger and K. M. Watson, Collision Theory (Wiley, New York, 1964).

[29] D. C. Latimer, Phys. Rev. D 88, 063517 (2013).

[30] D. R. Lorimer and M. Kramer, Handbook of Pulsar Astronomy (Cambridge University Press, Cambridge, United Kingdom, 2005), Vol. 4.

[31] V. Anastassopoulos et al. (CAST Collaboration), Nat. Phys. 13, 584 (2017).

[32] S. Gardner and D. C. Latimer, Phys. Rev. D 82, 063506 (2010).

[33] L. Shao and B. Zhang, Phys. Rev. D 95, 123010 (2017).

[34] R. N. Manchester, G. B. Hobbs, A. Teoh, and M. Hobbs, Astron. J. 129, 1993 (2005).

[35] L. Chuzhoy and E. W. Kolb, J. Cosmol. Astropart. Phys. 07 (2009) 014.

[36] D. Dunsky, L. J. Hall, and K. Harigaya, J. Cosmol. Astropart. Phys. 07 (2019) 015.

[37] L. M. Krauss and B. Chaboyer, Science 299, 65 (2003).

[38] J. M. Yao, R. N. Manchester, and N. Wang, Astrophys. J. 835, 29 (2016). 
[39] D. Foreman-Mackey, D. W. Hogg, D. Lang, and J. Goodman, Publ. Astron. Soc. Pac. 125, 306 (2013).

[40] S. Chatterjee et al., Nature (London) 541, 58 (2017).

[41] M. Pilia et al., Astron. Astrophys. 586, A92 (2016).

[42] S. Tremaine and J. E. Gunn, Phys. Rev. Lett. 42, 407 (1979).

[43] Y. Ali-Haïmoud, J. Chluba, and M. Kamionkowski, Phys. Rev. Lett. 115, 071304 (2015).

[44] J. B. Munoz and A. Loeb, Nature (London) 557, 684 (2018).

[45] T. R. Slatyer and C.-L. Wu, Phys. Rev. D 98, 023013 (2018).

[46] M. M. Ivanov, Y. Y. Kovalev, M. L. Lister, A. G. Panin, A. B. Pushkarev, T. Savolainen, and S. V. Troitsky, J. Cosmol. Astropart. Phys. 02 (2019) 059.

[47] D. Harari and P. Sikivie, Phys. Lett. B 289, 67 (1992).

[48] G. Sigl and P. Trivedi, arXiv:1811.07873.

[49] T. Fujita, R. Tazaki, and K. Toma, Phys. Rev. Lett. 122, 191101 (2019).

[50] A. D. Plascencia and A. Urbano, J. Cosmol. Astropart. Phys. 04 (2018) 059.

[51] I. Obata, T. Fujita, and Y. Michimura, Phys. Rev. Lett. 121, 161301 (2018).
[52] D. Ejlli, Nucl. Phys. B935, 83 (2018).

[53] A. Arvanitaki, S. Dimopoulos, and K. Van Tilburg, Phys. Rev. X 8, 041001 (2018).

[54] S. Weinberg, Phys. Rev. 126, 1899 (1962).

[55] W. Yan et al., Astrophys. Space Sci. 335, 485 (2011).

[56] J. L. Han, R. N. Manchester, A. G. Lyne, G. J. Qiao, and W. van Straten, Astrophys. J. 642, 868 (2006).

[57] T. Kobayashi, R. Murgia, A. De Simone, V. Irsi, and M. Viel, Phys. Rev. D 96, 123514 (2017).

[58] N. Bar, D. Blas, K. Blum, and S. Sibiryakov, Phys. Rev. D 98, 083027 (2018).

[59] D. J. E. Marsh and J. C. Niemeyer, Phys. Rev. Lett. 123, 051103 (2019).

[60] A. Payez, C. Evoli, T. Fischer, M. Giannotti, A. Mirizzi, and A. Ringwald, J. Cosmol. Astropart. Phys. 02 (2015) 006.

[61] M. Kramer and B. Stappers, Proc. Sci., AASKA14 (2015) 036.

[62] L. Shao et al., Proc. Sci., AASKA14 (2015) 042.

[63] P. Bull et al., arXiv:1810.02680.

[64] T. Liu, G. Smoot, and Y. Zhao, arXiv:1901.10981. 